\title{
Considerando a Motivação dos Estudantes em Experiências de Ensino-Aprendizagem de Computação
}

\author{
Bianca L. Santana ${ }^{1}$, Luis Gustavo J. Araujo ${ }^{1}$, \\ Roberto A. Bittencourt ${ }^{1}$ \\ ${ }^{1}$ UEFS - Universidade Estadual de Feira de Santana \\ Av. Transnordestina, s/n, Novo Horizonte \\ Feira de Santana - BA, Brasil - 44036-900 \\ \{biancasantana.ls, luisaraujo.ifba\}@gmail.com, roberto@uefs.br
}

\begin{abstract}
Several papers on programming learning investigate learning difficulties, measure learned skills, or present new teaching approaches, but little is investigated on student motivation to acquire these skills or how they face difficulties in these approaches. Motivation is hard to investigate because it is abstract and particular to each student, since each person has different goals when learning. On the other hand, several theories and models can be used to design and evaluate learning experiences from the point of view of motivation. One of these is the ARCS Model, which includes sets of strategies to increase the motivational appeal of instruction. Validated ARCS-based assessment tools can be used in a wide variety of courses. In this work, we present concepts and reflections on motivation, especially on the ARCS model and its theoretical bases. We illustrate the use of these concepts and models from our previous work. We seek to encourage the community to consider motivation in the design and evaluation of the teaching-learning process in computing.
\end{abstract}

Resumo. Diversos trabalhos em aprendizagem de programação investigam dificuldades de aprendizagem, mensuram competências aprendidas ou apresentam novas abordagens de ensino, mas pouco se investiga sobre a motivação dos estudantes para adquirir estas competências ou como eles encaram as dificuldades no decorrer destas abordagens. Motivação é difícil de se investigar por ser abstrato e particular a cada estudante, já que cada um possui objetivos diferentes ao aprender algo. Por outro lado, diversas teorias e modelos podem ser utilizadas para projetar ou avaliar experiências de aprendizagem sob o ponto de vista da motivação. Um destes é o Modelo ARCS, que inclui estratégias para aumentar o apelo motivacional da instrução. Instrumentos validados de avaliação baseados no Modelo ARCS podem ser utilizados em uma ampla variedade de formatos de cursos. Neste trabalho, apresentamos conceitos e reflexões sobre motivação, em especial sobre o Modelo ARCS e suas bases teóricas. Ilustramos o uso destes conceitos e modelo a partir de nossos trabalhos prévios. Buscamos incentivar a comunidade a considerar a motivação no design e avaliação do processo de ensino-aprendizagem de computação.

\section{Introdução}

A motivação lida com o comportamento humano e envolve processos que podem despertar um determinado comportamento e permitir que ele persista [Wlodkowski 1978]. É 
VII Congresso Brasileiro de Informática na Educação (CBIE 2018)

Anais dos Workshops do VII Congresso Brasileiro de Informática na Educação (WCBIE 2018)

natural que pessoas diferentes se sintam motivadas de maneiras diferentes. No contexto educacional, a motivação do estudante é um fator importante no processo de aprendizagem. Um estudante pode se sentir motivado pela vontade de aprender algum assunto, pelo simples medo do fracasso ou porque enxerga aquela disciplina como uma etapa a ser vencida no curso. Estudantes motivados por razões diferentes podem apresentar abordagens de estudo diferentes [Jenkins 2001].

O modo como algo é ensinado pode contribuir para aumentar ou reduzir a motivação dos estudantes. Diversos trabalhos buscam entender a motivação dos estudantes em disciplinas e cursos de computação. Jenkins (2001) realiza um estudo sobre a motivação em aprender a programar, definindo tipos de motivação apresentadas pelos estudantes. Bergin e Reilly (2005) discutem o papel da motivação em cursos de programação orientada a objetos, apontando relações explícitas entre o tipo de motivação apresentada pelo estudante e o nível de programação que ele consegue alcançar durante o curso. Nikula et al. (2011) relatam como um curso de programação, com altos índices de desistência e reprovação, teve suas taxas melhoradas em mais de $50 \%$ pela promoção de alterações focadas no aumento da motivação dos estudantes.

Como professores não podem se dar ao luxo de acreditar que seus estudantes estão motivados em aprender o que está sendo ensinado, existe a necessidade de se conceber cursos com materiais e metodologias que aumentem o apelo motivacional da instrução. Além disto, embora a motivação seja um conceito abstrato e, portanto, difícil de medir, é preciso saber como entender e avaliar os fatores motivacionais em um grupo.

Existem diversos modelos e teorias sobre motivação que podem ser empregadas no processo de ensino-aprendizagem, tanto para projetar experiências de aprendizagem quanto para avaliar a eficácia destas. Um destes é o Modelo de Atenção, Relevância, Confiança e Satisfação (ARCS), que inclui estratégias utilizadas para aumentar o apelo motivacional instrucional. ARCS é composto por quatro categorias conceituais que suprem vários dos conceitos e variáveis específicas que caracterizam a motivação humana [Keller 1987].

Neste trabalho, são apresentados conceitos e reflexões sobre o tema da motivação e do modelo ARCS. Também apresentamos brevemente duas de nossas experiências em projetar abordagens de ensino-aprendizagem de programação para diversas audiências: uma disciplina de Introdução à Ciência da Computação para Engenharia Civil e duas disciplinas de Programação para Cursos Técnicos de Informática. Com o intuito de aumentar a participação e engajamento dos estudantes, utilizamos as diretrizes e instrumentos de avaliação do modelo ARCS. Os resultados obtidos nos permitiram identificar a influência que nossas práticas de ensino exercem sobre a motivação dos estudantes. Com este artigo, visamos incentivar estudos teóricos ou empíricos que considerem a motivação durante o processo de ensino-aprendizagem em cursos de computação.

\section{Motivação}

Motivação é uma palavra que descreve processos que podem despertar um comportamento, dar propósito a ele, e permitir que ele persista, além de levar a escolher um determinado comportamento [Wlodkowski 1978].

Diversas técnicas e estratégias motivacionais podem ser usadas por professores para fortalecer o desempenho dos estudantes e reforçar atitudes positivas em relação 
à aprendizagem [Wlodkowski 1978]. Entwistle (2014) também reconhece o papel da motivação no ensino dos estudantes. Para ele, a motivação descreve a quantidade de esforço colocado em uma atividade e em seu objetivo. Motivação afeta, mas também é afetada, pelo nível de desempenho e é influenciada pelas percepções das tarefas de ensino e aprendizagem. Ele também acredita que o nível de motivação de um estudante é uma reação às circunstâncias, que dependem da história pessoal e dos hábitos de pensamento e estudo da pessoa. As estratégias de motivação afetam o desempenho acadêmico dos estudantes em conjunto com estratégias de aprendizagem [Zimmerman 1990].

Existe um variado corpo de pesquisa sobre esse tema. Muitos conceitos e teorias psicológicas tentam explicar a motivação para aprender. Cook e Artino (2016) sumarizam as cinco teorias contemporâneas sobre motivação para aprender. i) Valor-Expectativa: pressupõe que as pessoas são motivadas a participar de uma atividade se perceberem que ela está ligada à satisfação de suas necessidades pessoais e se existe uma expectativa positiva de sucesso. ii) Atribuição: os estudantes criam explicações causais subconscientes para os resultados de um evento. Estas atribuições, que variam em termos de locus, estabilidade e controlabilidade, influenciam as emoções, que, por sua vez, impulsionam a motivação em tarefas futuras. iii) Sócio-Cognitiva: a aprendizagem humana e o desempenho resultam de interações recíprocas entre fatores pessoais, comportamentais e ambientais. Aparecem no escopo desta teoria, a crença de autoeficácia, quando o estudante crê que pode executar com sucesso os comportamentos necessários para produzir um resultado desejado, e a auto-regulação, como um processo cíclico em que os indivíduos usam feedback auto-gerado sobre sua aprendizagem ou desempenho para gerenciar sua busca de objetivos pessoais. $i v$ ) Orientação ao Objetivo: os estudantes tendem a se engajar em tarefas com preocupações de dominar o conteúdo, fazer melhor do que outros ou evitar o fracasso. Algumas destes objetivos estimulam o interesse e o aprendizado profundo, enquanto outros estão associados a melhores notas. v) Autodeterminação: a motivação intrínseca leva o estudante a agir puramente para satisfazer sua curiosidade ou desejo de domínio, enquanto a motivação extrínseca é impulsionada por valores sociais. Ações extrinsecamente motivadas podem se autodeterminar à medida que os valores se tornam integrados e internalizados.

\section{Modelo ARCS}

O modelo Atenção, Relevância, Confiança e Satisfação (ARCS) é fundamentado na teoria valor-expectativa [Keller 1987], embora tenha sido atualizado por influência da teoria sócio-cognitiva [Keller 2009]. ARCS contém quatro categorias conceituais que suprem muitos dos conceitos e variáveis específicas que caracterizam a motivação humana. A categoria de Atenção traz diretrizes para obter, manter e dirigir a atenção dos estudantes para os estímulos apropriados. A categoria Relevância oferece diretrizes para que a instrução pareça relevante não só para as oportunidades de carreira do estudante, mas também busca aumentar a sensação de relevância do curso através do modo como ele é ensinado. A categoria Confiança traz diretrizes para formar no estudante a impressão de que algum nível de sucesso é possível se esforço for exercido. A categoria Satisfação incorpora pesquisas e práticas que ajudam as pessoas a se sentirem bem com suas realizações.

O ARCS incorpora um processo sistemático de design, chamado design motivacional, que pode ser usado na criação de materiais e planejamento de aulas [Keller 2009]. São quatro etapas pre-estabelecidas: definir, projetar, desenvolver e avaliar. A Tabela 1 
VII Congresso Brasileiro de Informática na Educação (CBIE 2018)

Anais dos Workshops do VII Congresso Brasileiro de Informática na Educação (WCBIE 2018)

Tabela 1. Etapas do Design Motivacional [Keller 2009].

\begin{tabular}{ll|l}
\hline \multicolumn{2}{l|}{ Etapas } & \multicolumn{2}{l}{ Descrição } \\
\hline $\mathbf{1}$ & Definir & $\begin{array}{l}\text { Classificar o problema motivacional a ser resolvido e } \\
\text { preparar objetivos motivacionais. }\end{array}$ \\
\hline $\mathbf{2}$ & Projetar & $\begin{array}{l}\text { Criar uma lista de potenciais estratégias para os } \\
\text { objetivos motivacionais definidos. }\end{array}$ \\
\hline $\mathbf{3}$ & Desenvolver & Criar os materiais necessários. \\
\hline $\mathbf{4}$ & Avaliar & $\begin{array}{l}\text { Julgar as consequências motivacionais do que foi proposto em } \\
\text { termos de medidas diretas de persistência, intensidade de esforço, } \\
\text { emoção e atitude. }\end{array}$ \\
\hline
\end{tabular}

descreve cada uma destas etapas. A etapa de definição prevê a análise da audiência para identificar lacunas motivacionais.

A maioria dos estudos sobre motivação em aprender centra-se na descrição das estratégias motivacionais aplicadas sem o apoio de dados empíricos [Huang et al. 2006]. Para diagnosticar problemas motivacionais em materiais instrucionais, Keller (1993) desenvolveu um instrumento de medição chamado Instructional Material Motivation Survey (IMMS). Neste questionário, há 36 declarações em escala de Likert e todas elas foram desenvolvidas de acordo com componentes individuais do ARCS. Os construtos do IMMS estão mais relacionados a materiais autodirigidos, mas, mesmo assim, oferecem base para avaliação em outros formatos de cursos presenciais. Além do IMMS, Keller (2009) também propôs o questionário Course Interest Survey (CIS), com o objetivo de mensurar a motivação dos estudantes em relação a um determinado curso ou disciplina presencial. No CIS, há 34 declarações em escala de Likert. Assim como o IMMS, o CIS foi elaborado para estar em correspondência com a base teórica representada pelos conceitos motivacionais e teorias que compõem o modelo ARCS.

\section{Nossas Experiências com o Modelo ARCS}

O design motivacional do ARCS considera que entender a motivação dos estudantes é o principal fator para proporcionar uma experiência de aprendizagem significativa. Mesmo sendo impossível entender os objetivos e o comportamento de cada estudante, é importante considerar a audiência para a qual será ministrado o curso ou até mesmo identificar tipos de estudantes dentro de uma dada audiência. A seguir, descrevemos como nós utilizamos o modelo ARCS para entender a motivação de estudantes em aprender a programar.

\subsection{Experiência na Educação Superior}

Em nossa instituição, os cursos de Engenharia Civil e Engenharia de Alimentos oferecem uma disciplina chamada Introdução à Ciência da Computação (ICC), cujo foco é o ensino de programação e de tópicos de ciência da computação. Os professores relatam haver pouco engajamento nas turmas de ICC e consideram difícil comunicar o valor da programação para os estudantes. Por isso, concebemos uma abordagem de ensino-aprendizagem de programação para esta audiência, e avaliamos sua influência na motivação dos estudantes através de dois estudos de caso. A abordagem proposta combina o uso do ambiente Scratch em um contexto de criação de jogos, a linguagem Python associada à biblioteca Turtle Graphics e a manipulação de imagens por meio do IDE JES.

As diretrizes e os instrumentos do modelo ARCS foram utilizados no planejamento, no processo de levantamento de dados e em análises posteriores. Neste artigo, 
VII Congresso Brasileiro de Informática na Educação (CBIE 2018)

Anais dos Workshops do VII Congresso Brasileiro de Informática na Educação (WCBIE 2018)

nos limitamos a exemplificar como o modelo ARCS pode ser empregado neste tipo de estudo, embora detalhes específicos sobre a abordagem e resultados de nossa experiência estão disponíveis em outros trabalhos [Santana et al. 2017, Santana and Bittencourt 2018, Santana et al. 2018].

Inicialmente, realizamos um estudo de caso com uma turma de ICC para Engenharia Civil onde aplicamos a primeira versão de nossa abordagem. Foram 36 estudantes participantes, sendo 27 do sexo masculino e 9 do feminino. O objetivo era identificar os pontos fortes e os pontos fracos de nossa abordagem a fim de propor melhorias para uma nova versão da abordagem e dos próprios instrumentos de medição. A partir da análise dos resultados obtidos, promovemos mudanças na abordagem e voltamos a aplicá-la em um segundo estudo de caso com outra turma de ICC para Engenharia Civil, analisando novamente sua influência na motivação dos estudantes. No segundo estudo de caso, contamos com 37 estudantes participantes, sendo 25 do sexo masculino e 12 do feminino.

A disciplina foi organizada em três unidades, cada uma utilizando ferramentas e contextos metodológicos diferentes. Avaliamos cada unidade com o questionário IMMS, entrevistas e observações. Ao final da disciplina, aplicamos o questionário CIS e realizamos entrevistas com questões elaboradas especificamente para identificar aspectos que influenciam individualmente na atenção, relevância, confiança e satisfação dos estudantes.

Cada construto dos questionários IMMS e CIS está relacionado a uma das quatro categorias do ARCS. No IMMS, dos 36 construtos, 12 são da categoria atenção, 9 de relevância, 9 de confiança e 6 de satisfação. Deste modo, a partir dos resultados dos questionários, é possível definir um escore em escala de Likert através da média aritmética das respostas de cada indivíduo para uma dada categoria (convertendo a escala nominal em numérica e invertendo a escala para as questões fraseadas na forma negativa). Este escore é uma nova variável aleatória da qual extraímos estatísticas descritivas de concentração e dispersão, como média e desvio-padrão.

Como aplicamos o questionário IMMS em cada unidade, computamos médias para os níveis de atenção, relevância, confiança e satisfação para cada unidade. Estes resultados nos permitiram fazer testes de hipótese sobre os níveis de atenção, relevância, confiança e satisfação ao longo das unidades. A partir daí, comparamos individualmente cada categoria. Empregamos um processo semelhante em cada uma delas, mas, aqui, vamos descrever o processo apenas para a categoria de Atenção. A Figura 1 exibe os resultados de Atenção para a primeira unidade. Para cada unidade, adaptamos os construtos à realidade do contexto utilizado. Três construtos que não puderam ser adaptados ao nosso contexto foram descartados.

A Figura 2 apresenta um gráfico box-plot dos escores de atenção e um diagrama de barras de erros para as médias do escore da categoria Atenção no segundo estudo de caso realizado. A partir dos dados brutos dos questionários, calculamos o escore para cada participante e geramos as estatísticas descritivas. Os resultados demonstram que o nível de atenção cai no decorrer das três unidades. A partir das estatísticas descritivas, e de resultados positivos para testes de normalidade no conjunto de dados, realizamos um teste $\mathrm{t}$ de Student em cada um dos pares possíveis, permitindo-nos confirmar ou não a significância da variação da atenção entre dadas duas unidades. No caso da categoria Atenção, o teste nos trouxe a constatação de que só houve diferença significativa nos 
VII Congresso Brasileiro de Informática na Educação (CBIE 2018)

Anais dos Workshops do VII Congresso Brasileiro de Informática na Educação (WCBIE 2018)

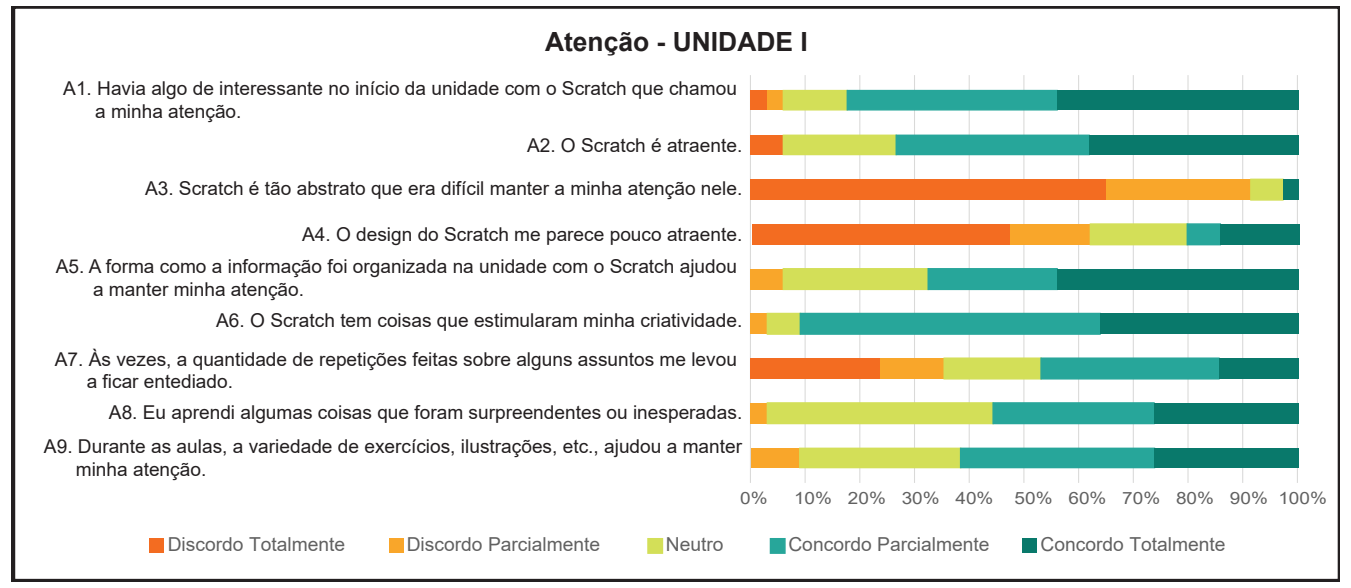

Figura 1. Resultados para a categoria Atenção, na primeira unidade, com o questionário IMMS.

níveis de atenção entre a primeira e a terceira unidades.

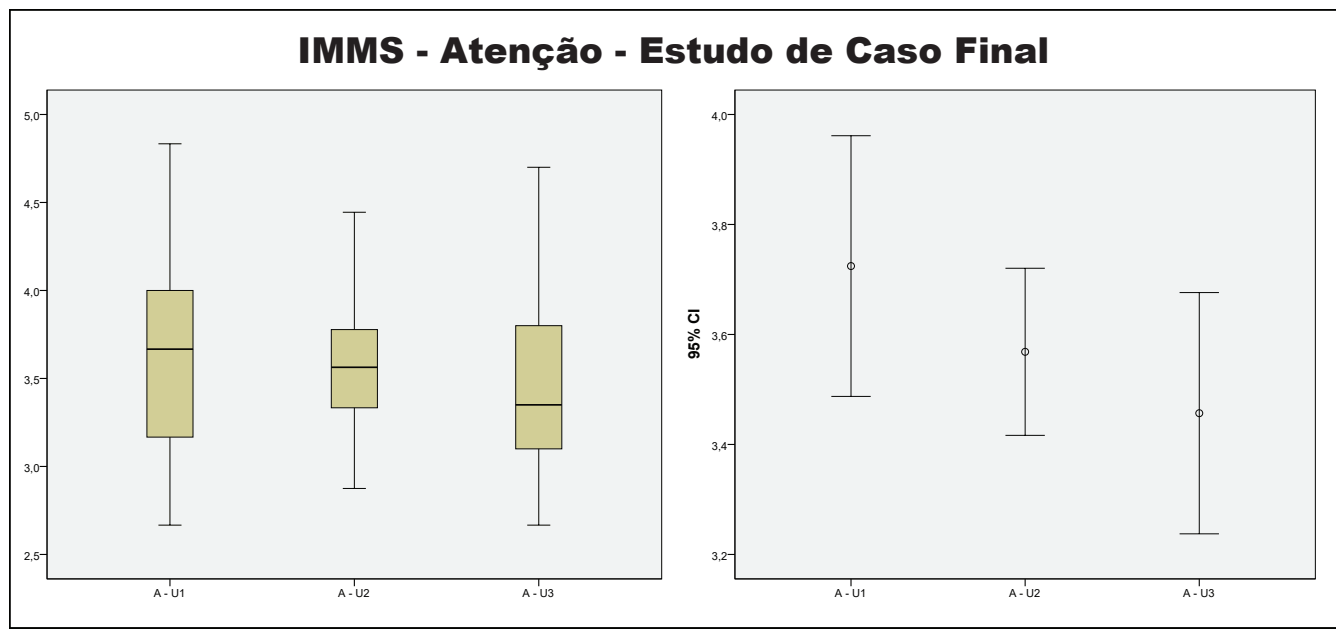

Figura 2. Box-plot e diagrama de barra de erros para a categoria Atenção em cada uma das unidades.

Para os dados qualitativos, provenientes de observações das aulas e entrevistas, fizemos a sua codificação, inicialmente de maneira livre (codificação aberta). Os códigos emergentes foram agrupados e relacionados a uma ou mais características de nossa abordagem, como postura do professor, formato de aulas, ferramentas, linguagens, entre outras. Numa etapa final, associamos cada um dos fatores de nossa abordagem a uma ou mais variáveis contidas nas categorias do ARCS (codificação axial).

De acordo com o modelo ARCS, a categoria Atenção possui três variáveis principais: Perceptual Arousal, Inquiry Arousal, e Variabilidade. Perceptual Arousal se refere às atitudes que visam captar o interesse do estudante, Inquiry arousal se refere às atitudes que podem estimular uma atitude de indagação no estudante, enquanto Variabilidade se refere às atitudes que visam manter a atenção do estudante [Keller 2009]. Frequentemente, os extratos qualitativos indicavam um determinado fator de nossa abordagem que contribui, positiva ou negativamente, para estas variáveis e, consequentemente, influencia os níveis de atenção. 
VII Congresso Brasileiro de Informática na Educação (CBIE 2018)

Anais dos Workshops do VII Congresso Brasileiro de Informática na Educação (WCBIE 2018)

Percebemos em nossas observações que quando o professor utiliza de maneira alternada em uma mesma aula diversos recursos, como explicações no quadro, slides, e desenvolvimentos de projetos, a turma fica menos dispersa, e isso influi positivamente na variável de variabilidade. Contrastando ainda esta suposição com os resultados quantitativos, percebemos que, na primeira unidade, onde houve mais registros desse tipo de atitude, o nível de atenção foi ligeiramente maior. Desta maneira, identificamos como os principais aspectos de nossa abordagem influenciam na motivação dos estudantes. A Figura 3 apresenta os fatores que influenciam em cada uma das variáveis de atenção, e consequentemente, nesta categoria. Utilizamos este processo para criar um quadro para cada uma das categorias e, posteriormente, compilamos todos os resultados em um quadro geral.

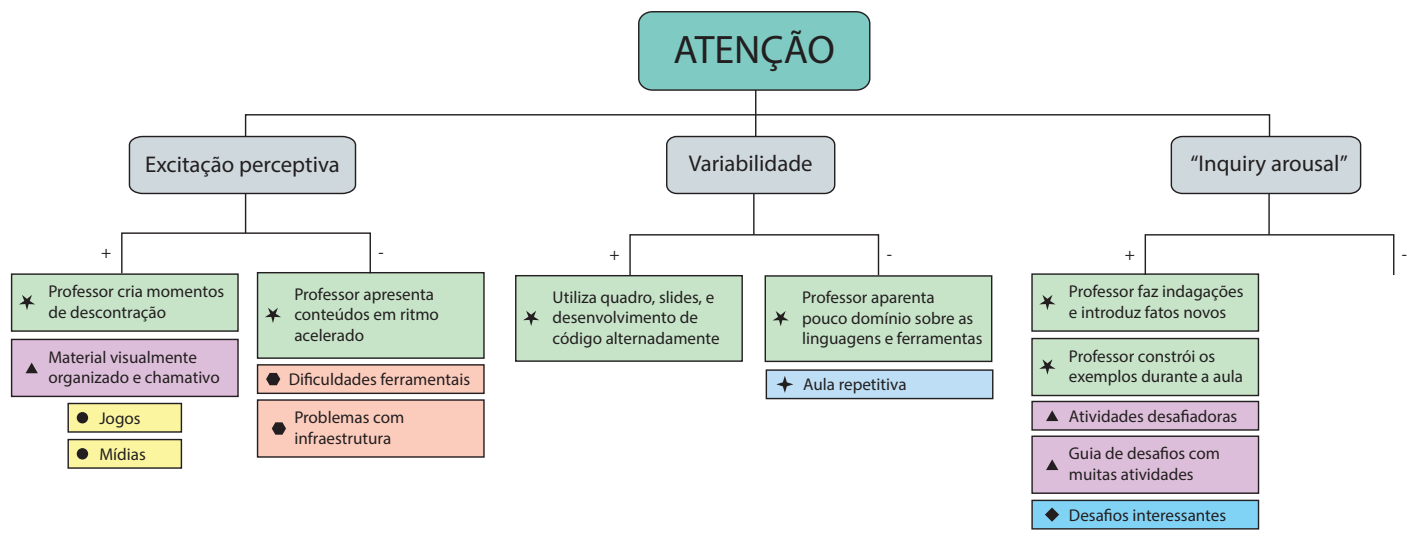

Figura 3. Fatores de nossa abordagem que influenciam na atenção dos estudantes.

O questionário CIS também foi utilizada em nossa pesquisa. Utilizamos este questionário para realizar análises entre as categorias. Por exemplo, comparamos o nível de atenção com o de relevância. Também aplicamos o CIS com uma turma de ICC que não utilizou nossa abordagem e utilizamos os resultados para propor uma comparação entre os níveis de cada categoria nas duas abordagens.

\subsection{Experiência na educação profissional}

Os cursos técnicos em Informática da Rede Estadual de Educação Profissional do Estado da Bahia fazem parte do Eixo de Informação e Comunicação e possuem um currículo formado por três grupos de disciplinas: Base Nacional Comum (BNC), Formação Técnica Geral (FTG) e Formação Técnica Específica (FTE). Os componentes curriculares da FTE relacionam-se com a área dos cursos técnicos. Estas disciplinas são distribuídas, na modalidade de ensino integrado, ao longo de quatro anos. Nos cursos técnicos em Informática, as disciplinas de programação fazem parte do grupo FTE e costumam sofrer de problemas de evasão e reprovação elevadas.

Assim como na experiência na Educação Superior, realizamos um estudo de caso preliminar com uma primeira versão de nossa abordagem (no caso, testada com uma turma do nono ano do ensino fundamental) com os mesmos objetivos: identificar os pontos fortes e os pontos fracos da abordagem a fim de propor melhorias 
VII Congresso Brasileiro de Informática na Educação (CBIE 2018)

Anais dos Workshops do VII Congresso Brasileiro de Informática na Educação (WCBIE 2018)

[Araujo et al. 2018a]. Após a análise deste estudo de caso, levamos a abordagem melhorada para a Educação Profissional e realizamos mais dois estudos de caso em disciplinas de cursos técnicos em Informática: Lógica e Técnica de Programação, no $2^{\circ}$ ano, e Linguagem de Programação, no $3^{\circ}$ ano. Os estudos de caso foram realizados em duas escolas da Rede Estadual do município de Feira de Santana, Bahia. Informações mais detalhadas sobre estas experiências podem ser encontradas em outros artigos [Araujo et al. 2017, Araujo et al. 2018b]. Aqui, nos limitamos a ilustrar como o modelo ARCS pode ser empregado neste tipo de estudo.

$\mathrm{O}$ ano letivo das disciplinas foi dividido em três blocos contextualizados, utilizando contextos como criação de figuras, jogos e efeitos em imagens. Além disso, variamos o uso de ferramentas: Scratch, JES e PPlay. Assim como a experiência apresentada anteriormente, avaliamos cada unidade com o questionário IMMS, entrevistas e observações, além de avaliações e diário de bordo. O questionário CIS foi aplicado ao término da disciplina. O cômputo dos escores das categorias motivacionais foi realizado do mesmo modo que no estudo de caso na educação superior. Assim, calculamos o escore de cada categoria do ARCS e realizamos, dada a pequena amostra, testes de hipótese de Wilcoxon para verificar se houve mudança significativa entre os blocos. Realizamos, ainda, os mesmos testes de hipótese de para cada construto individual do IMMS e do CIS, além de computar a correlação de Spearman entre as quatro dimensões do ARCS e entre cada uma delas e o aprendizado de conceitos de programação.

Os dados qualitativos, provenientes de observações, diários de bordo e entrevistas, assim como no estudo anterior, foram codificados inicialmente com codificação aberta. Os códigos emergentes foram agrupados e relacionados às categorias específicas, visando iluminar os resultados quantitativos. Em seguida, sobre os aspectos da motivação presentes nos dados, buscamos agrupar os achados de acordo com as categorias do modelo ARCS.

Como o objetivo de triangular os achados qualitativos e quantitativos, construímos, a partir dos resultados, mapas mentais para cada categoria do ARCS, mapas estes que ajudam a compreender como a nossa abordagem influencia na motivação dos estudantes. Realizamos este procedimento para as quatro categorias do ARCS, para os resultados dos três estudos de caso, mas apresentamos aqui apenas a dimensão de Relevância de um dos estudos de caso para fins de ilustração (ver Figura 4).

Assim, percebemos que alguns fatores relacionados à abordagem, como a linguagem Python, os ambientes JES e PPlay, os contextos de Jogos e Imagens, além dos materiais de aula e de elementos pedagógicos como o ensino em espiral, colaboração entre os estudantes e a mediação, contribuem para o aumento da relevância, como pode-se observar na Figura 4.

\section{Conclusões}

Neste trabalho, apresentamos conceitos e reflexões sobre o tema da motivação e sobre o modelo ARCS. Também apresentamos relatos sobre como utilizamos este modelo para entender a motivação de estudantes de diferentes audiências em aprender a programar. As diretrizes e os instrumentos de avaliação do modelo ARCS nos ajudaram nas avaliações de nossas abordagens de ensino-aprendizagem de programação, tanto para estudantes de graduação que não são da área de TI, como para estudantes de cursos técnicos de 


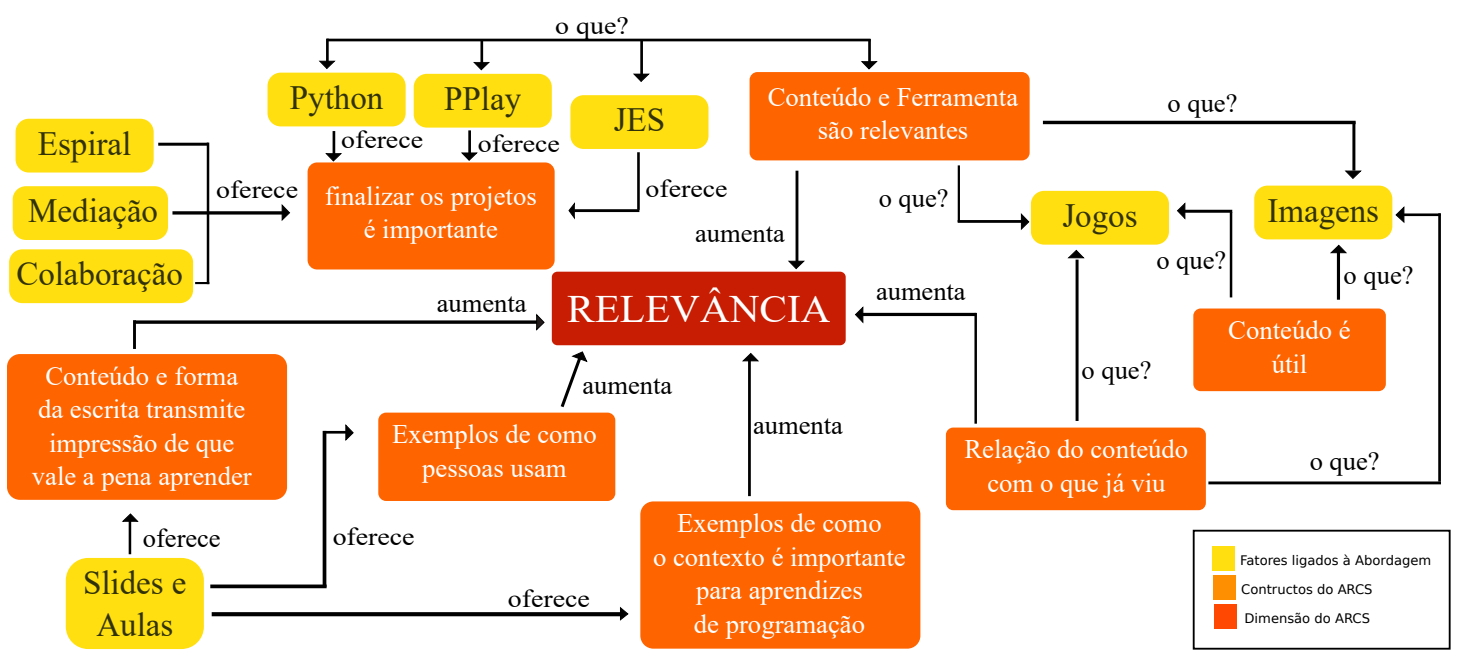

Figura 4. Fatores de nossa abordagem que influenciam na Relevância para os estudantes.

Informática. Os resultados obtidos nos permitiram identificar a influência que nossas práticas de ensino exercem sobre a motivação dos estudantes e, consequentemente, propor melhorias para aumentar a participação e o engajamento dos estudantes nestas disciplinas.

Em nossas experiências, o modelo ARCS demonstrou ser um modelo simples e adaptável para diferentes ambientes de aprendizagem. Os construtos dos questionários IMMS e CIS podem ser facilmente adaptados de acordo com o contexto. O fato de estes construtos já estarem previamente relacionados a uma categoria simplifica o processo de análise. Além disto, as diretrizes e o aporte teórico subjacente ao modelo ARCS nos deram bases suficientes para interpretação e sintetização dos resultados obtidos. Finalmente, as diferentes maneiras de sintetizar e apresentar os resultados sobre motivação dependem da variedade de dados disponíveis e dos interesses específicos e estilo dos pesquisadores, provendo flexibilidade na análise, como pôde ser observado nos dois estudos apresentados.

Apesar da subjetividade do tema da motivação, através de nossos estudos, conseguimos identificar práticas que podem aumentar a motivação dos estudantes e práticas que podem diminuí-la. Promovemos estudos robustos, com coleta de dados qualiquantitativos, mas temos razões suficientes para acreditar que a simples aplicação de um dos questionários, CIS ou IMMS, já traz insights relevantes sobre os níveis de atenção, relevância, confiança e satisfação dos estudantes. Nosso principal objetivo com este artigo é incentivar a comunidade a considerar o tema da motivação no design e avaliação do processo de ensino-aprendizagem em disciplinas de computação, especialmente em pensamento computacional ou em programação. Acreditamos na importância dos aspectos motivacionais no processo de aprendizagem e no potencial impacto positivo para a comunidade em considerá-los em seus trabalhos.

\section{Referências}

Araujo, L. G. J., Bittencourt, R. A., and Santos, D. M. B. (2017). Uma abordagem contextualizada para o ensino de programação na educação profissional em informática. In III Workshop de Ensino em Pensamento Computacional, Algoritmos e Programação. 
VII Congresso Brasileiro de Informática na Educação (CBIE 2018)

Anais dos Workshops do VII Congresso Brasileiro de Informática na Educação (WCBIE 2018)

Araujo, L. G. J., Bittencourt, R. A., and Santos, D. M. B. (2018a). An analysis of a mediabased approach to teach programming to middle school students. In SIGCSE 2018 49th ACM Technical Symposium on Computer Science Education. ACM.

Araujo, L. G. J., Bittencourt, R. A., and Santos, D. M. B. (2018b). Contextualized spiral learning of computer programming in brazilian vocational secondary education. In FIE 2018 - 48th Annual Frontiers In Education Conference.

Bergin, S. and Reilly, R. (2005). The influence of motivation and comfort-level on learning to program.

Cook, D. A. and Artino, A. R. (2016). Motivation to learn: an overview of contemporary theories. Medical Education, 50(10):997-1014.

Entwistle, N. (2014). Motivation and approaches to learning: Motivating and conceptions of teaching. In Motivating students, pages 25-34. Routledge.

Huang, W., Huang, W., Diefes-Dux, H., and Imbrie, P. K. (2006). A preliminary validation of attention, relevance, confidence and satisfaction model-based instructional material motivational survey in a computer-based tutorial setting. British Journal of Educational Technology, 37(2):243-259.

Jenkins, T. (2001). The motivation of students of programming.

Keller, J. (1993). Motivation by design. Unpublished manuscript, Florida State University, Florida.

Keller, J. M. (1987). Development and use of the arcs model of instructional design. Journal of instructional development, 10(3):2-10.

Keller, J. M. (2009). Motivational design for learning and performance: The ARCS model approach. Springer Science \& Business Media.

Nikula, U., Gotel, O., and Kasurinen, J. (2011). A motivation guided holistic rehabilitation of the first programming course. ACM Trans. Comp. Education (TOCE), 11(4):24.

Santana, B. L. and Bittencourt, R. A. (2018). Increasing motivation of cs1 non-majors through an approach contextualized by games and media. In FIE 2018 - 48th Annual Frontiers In Education Conference.

Santana, B. L., Figuerêdo, J. S. L., and Bittencourt, R. A. (2017). Motivação de estudantes non-majors em uma disciplina de programação. In WEI 2017 - XXV Workshop sobre Educação em Computação.

Santana, B. L., Figueredo, J. S. L., and Bittencourt, R. A. (2018). Motivation of engineering students with a mixed-contexts approach to introductory programming. In FIE 2018 - 48th Annual Frontiers In Education Conference.

Wlodkowski, R. J. (1978). Motivation and teaching: A practical guide.

Zimmerman, B. J. (1990). Self-regulated learning and academic achievement: An overview. Educational psychologist, 25(1):3-17. 